\title{
A DNA-Based Approach to the Carbon Nanotube Sorting Problem
}

\author{
Xiaomin Tu and Ming Zheng（ $\bowtie ）$ \\ DuPont Central Research and Development, Wilmington, DE 19880, USA \\ Received: 6 June 2008/ Revised: 1 July 2008/Accepted: 7 July 2008 \\ CTsinghua Press and Springer-Verlag 2008. This article is published with open access at Springerlink.com
}

\begin{abstract}
Carbon nanotube sorting, i.e., the separation of a mixture of tubes into different electronic types and further into species with a specific chirality, is a fascinating problem of both scientific and technological importance. It is one of those problems that are easy to describe but difficult to solve. Single-stranded DNA forms stable complexes with carbon nanotubes and disperses them effectively in water. A particular DNA sequence of alternating guanine $(\mathrm{G})$ and thymine $(\mathrm{T})$ nucleotides $\left((\mathrm{GT})_{n}\right.$, with $n=10$ to 45$)$ self-assembles into an ordered supramolecular structure around an individual nanotube, in such a way that the electrostatic properties of the DNA-carbon nanotube hybrid depend on tube structure, enabling nanotube separation by anion-exchange chromatography. This review provides a summary of the separation of metallic and semiconducting tubes, and purification of single $(n, m)$ tubes using the DNA-wrapping approach. We will present our current understanding of the DNA-carbon nanotube hybrid structure and separation mechanisms, and predict future developments of the DNA-based approach.
\end{abstract}

\section{KEYWORDS}

Carbon nanotube separation, DNA-wrapped carbon nanotubes, metallic and semiconducting tubes

\section{The carbon nanotube sorting problem}

Carbon nanotubes (CNTs) are a family of onedimensional tubular molecular structures with unique physical and chemical properties [1]. Conceptually, a single-walled CNT is a hollow cylinder formed by rolling up a graphene sheet made up of hexagonally bonded $\mathrm{sp}^{2}$ carbon atoms. The direction and magnitude of a roll-up vector, denoted by chiral indexes $(n, m)$, prescribe the chirality and diameter, respectively, of the resulting tube (Fig. 1(a)). These geometric parameters in turn determine the electronic band structure of the tube. Tremendous progress has been made in CNT synthesis. However, making CNTs with a particular structure still remains a major challenge in both fundamental studies and development of applications. The root cause of the problem is that the high temperature $\left(>500{ }^{\circ} \mathrm{C}\right)$ gas phase processes required for the synthesis cannot be sufficiently selective to favor one particular structure over others. The most selective synthesis processes reported to date yield one or two major CNT species in $20 \%-30 \%$ abundance, along with many other minor species of similar diameter [2-4]. Physically separating different $\mathrm{CNT}$ structures through a room temperature solution phase process is thus not only a fascinating scientific problem, but also has significant technological importance.

Manipulation of tubular molecular structures

Address correspondence to ming.zheng@usa.dupont.com

\section{算 Springer}


such as CNTs represents a new frontier in the broad field of molecular science. To solve the sorting problem, new tools, processes, and concepts have to be developed, which will contribute to the expansion of the knowledge base of molecular science. The availability of pure CNT species directly benefits fundamental physical and chemical studies of CNTs, as well as those applications that are sensitively dependent on tube structures. For those of us working on the CNT separation problem, each ( $n$, $m)$ structure has its unique aesthetic appeal. The ultimate solution to the CNT sorting problem should be a process through which each and every single $(n$, $m)$ species from a given mixture can be purified.
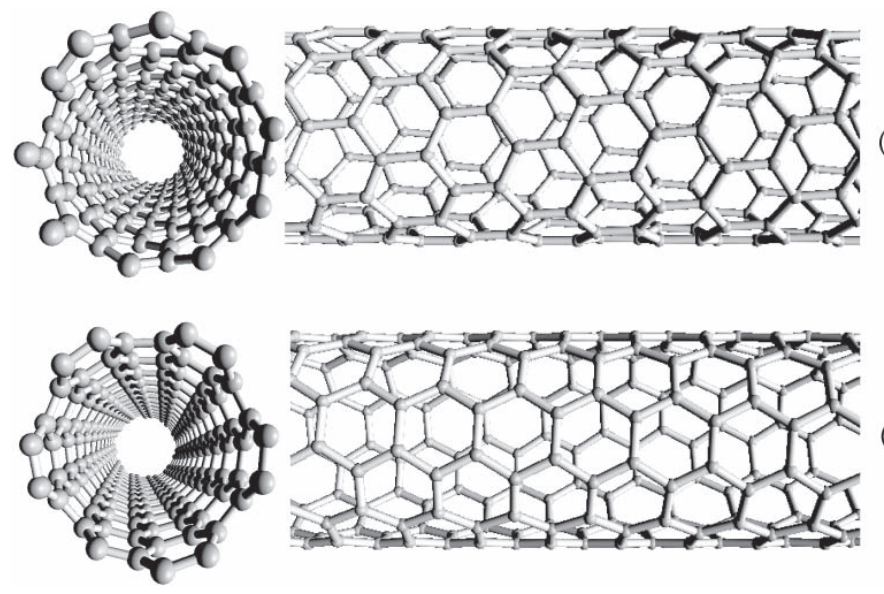

(a)

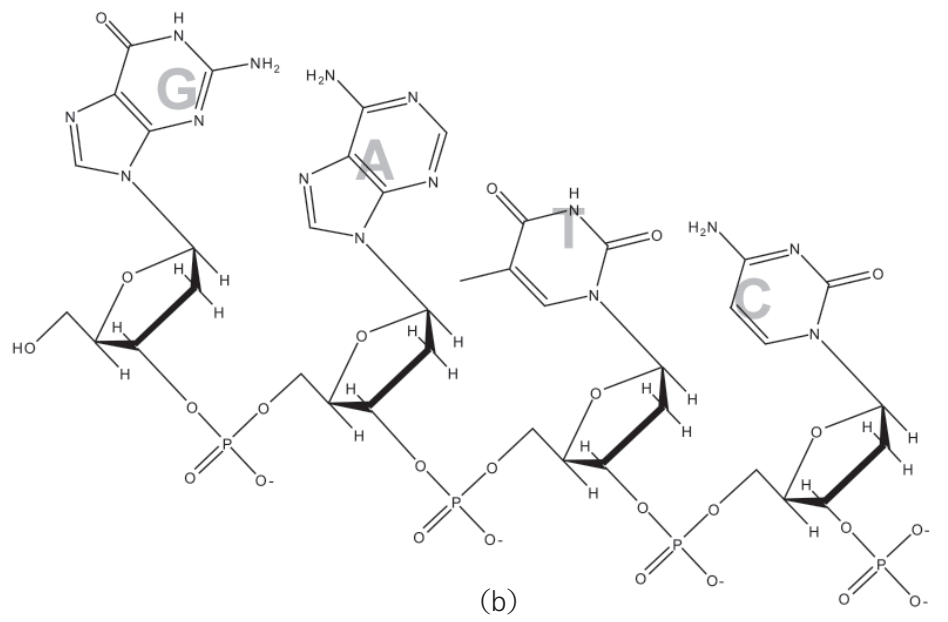

Figure 1 (a) Structures of two semiconductor single-walled carbon nanotubes $(9,1)$ and $(6,5)$. These two tubes have different chiralities but the same diameter. (From Ref. [7], used with permission) (b) A short oligonucleotide structure composed of guanine $(G)$, adenine $(A)$, thymine $(T)$, and cytosine $(C)$. Note that each nucleotide consists of a nitrogenous base, a deoxyribose, and a negatively charged phosphate group
Different degrees of CNT separation can be classified. A single-walled CNT can be either metallic or semiconducting, depending solely on its chiral index $(n, m)$. As a consequence of their different electronic structures, metallic and semiconducting tubes have distinct chemical reactivities, and different physical properties such as polarizability. Exploiting all these differences in order to separate the two types of tube constitutes one degree of separation. Within the same electronic type, tube diameter determines surface area per unit tube length, affecting such quantities as linear charge density and effective hydrodynamic size for dispersed tubes. Separation by diameter is therefore also conceptually feasible and constitutes another degree of separation. The most demanding task, in our opinion, is to separate two species of the same electronic type and same diameter but different chiralities (Fig. 1(a)). To a first order of approximation, these tubes have little difference in their electronic structures. The challenge in this type of separation is how to convert the minute difference into something macroscopically measurable and the DNA-based approach appears to be especially suited for this purpose.

The CNT separation problem has drawn considerable attention over the past few years. Many separation mechanisms have been devised and various degrees of separation demonstrated [5-16]. It is not our intention here to give a comprehensive review of each reported approach; interested readers should consult reviews published elsewhere [17, 18]. Nevertheless, we do want to comment briefly on a few representative approaches here. The first is the impressive work carried out by Hersam and his collaborators [11, 14]. They demonstrated clearly that metal/ semiconductor and diameter-based separation could be achieved with density gradient ultracentrifugation of CNTs dispersed by a combination of two surfactants, sodium dodecyl sulfate, and sodium cholate. Taking advantage of differential adsorption of octadecylamine (ODA) on CNTs, Maeda et al. demonstrated enrichment of metallic tubes after a dispersion- 
centrifugation process [12]. This work is related to an earlier study carried out by Papadimitrakopoulos and co-workers, in which ODA was used to suspend carboxylated CNTs in an organic solvent such as tetrahydrofuran (THF). The stronger binding affinity of ODA for semiconducting tubes in this case allowed their enrichment by sedimentation [9].

In the last six years or so, we have been developing chromatographic separation of DNAwrapped carbon nanotubes (DNA-CNT) [5-7, 19]. In this review, we will present the different degrees of CNT separation achieved so far by the DNA-based approach. We will also discuss some fundamental issues faced by the approach and the prospects for its future development.

\section{DNA-based separation}

\subsection{Scientific background}

The idea of using DNA (Fig. 1(b)) for CNT manipulation is not as exotic as it appears to be at first sight. Two separate lines of scientific inquiry leading to the idea can be traced. First, there have been many experimental studies of the interaction between the nitrogenous bases of DNA and various inorganic surfaces, among which graphite is most prevalent [20 -22]. Interestingly, these studies bear relevance to the understanding of molecular evolution and origin of life [22]. Two conclusions pertinent to our work can be drawn from these studies: (1) the four nitrogenous bases of DNA all have strong adsorption affinities to a graphite surface, but the magnitude varies; (2) DNA bases can assemble on a graphite surface into a monolayer through hydrogen bonding interactions, in registration with the underlying lattice structure. This is a direct consequence of the physical/chemical properties of DNA bases: they have planar aromatic structures, and their peripheral functional groups are arranged in such a way that a variety of hydrogen bonding geometries can be established. The structure of double stranded DNA best illustrates these properties: the $\pi-\pi$ stacking interaction between the planar bases provides stabilizing forces to the structure, and hydrogen bonding interactions further hold the two complementary strands together.
The second line of investigation that influenced our work is the in vitro evolution (or aptamer) technique developed in biochemical research [23]. Single-stranded DNA (ssDNA) forms a large molecular library. Structural varieties derived from the huge number of sequences ensure the existence of certain sequences that can recognize/bind any given small molecule targets. The in vitro evolution technique is a systematic way to find these sequences by using the tools of molecular biology.

\subsection{DNA-wrapped carbon nanotubes}

The ssDNA library offers intriguing possibilities for CNT binding. Without knowing how strong the DNA/CNT interaction could be, our initial thought was that depending on its sequence and structure, aromatic bases in an ssDNA may be able to form $\pi$-stacking interactions with the side-wall of CNTs. We therefore began our work by searching for CNT binding sequences following the standard in vitro evolution procedure. It was during this process that we were surprised to find strong DNA binding to CNTs [5]. This led to the idea of CNT dispersion by DNA. Not only ssDNA of almost any sequence, but also short double-stranded DNA, and total RNA extracted from bacteria can also disperse CNTs. Sonication is needed for effective dispersion, similar to the conditions required for CNT dispersion by many surfactants. DNA-CNT solutions are stable for months at room temperature. Removal of free DNA by either anion exchange column chromatography or nuclease digestion does not cause nanotube flocculation, indicating that DNA binding to carbon nanotubes is very strong. Evidence from atomic force microscopy (AFM), UV-vis-near IR absorption and fluorescence spectroscopy strongly suggest that DNA converts bundled CNT into individual tubes.

What is the structure of ssDNA on a CNT? AFM revealed periodic features along a DNA-dispersed $\mathrm{CNT}$, indicative of a helical wrapping structure of DNA around CNT. A crude molecular dynamics simulation, that did not include solvent, also suggested a helical structure, in which the bases are stacked onto the CNT surface and the sugarphosphate backbone is extended outward [5] (Fig. 2). Subsequent circular dichroism measurements on 


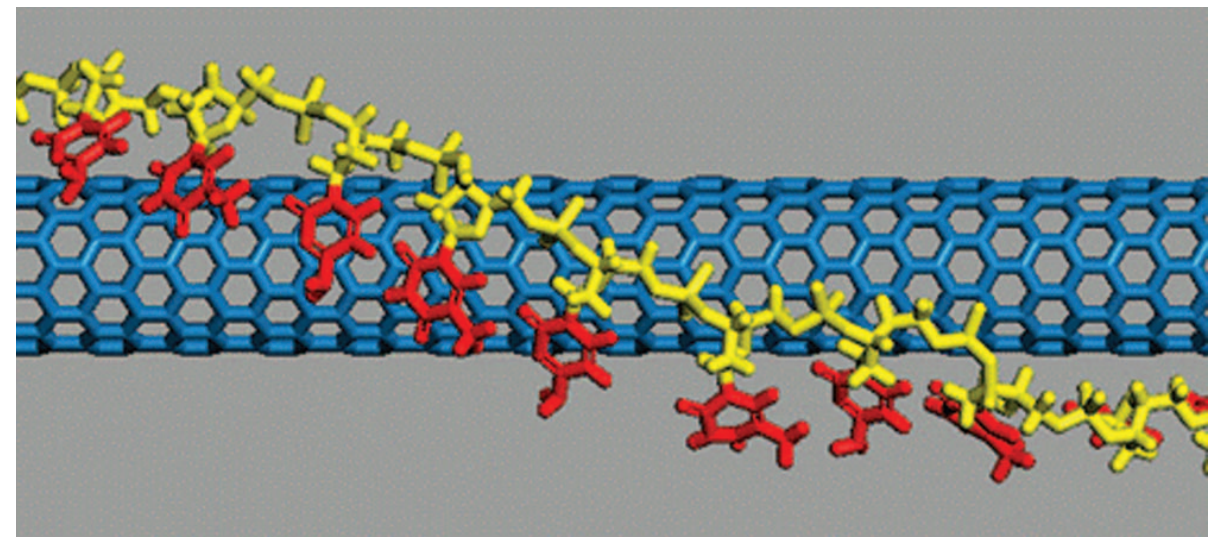

Figure 2 A structure model of DNA-wrapped carbon nanotube (Courtesy of Dr. Anand Jagota)

DNA-CNTs also found evidence consistent with the helical wrapping structure [24]. Recently, Johnson et al. revisited the issue with molecular dynamics simulations that did include the effect of aqueous solvents [25]. They observed a strong tendency for ssDNA to form a helical wrapping from the $3^{\prime}$ end to the $5^{\prime}$ end, and attributed the mechanism to electrostatic and torsional interactions within the sugar-phosphate backbone. Binding energies of DNA to CNT have also been examined by different levels of calculation [25-29]; all of them give a strong binding energy of $\sim 10 \mathrm{kT}$ per nucleotide or larger, with slight differences between the four nucleotides. The binding energy derives largely from the $\pi-\pi$ stacking interaction, but also has contributions from the sugar-phosphate backbone.

\subsection{Chromatographic separation of DNA-CNT}

The availability of DNA-CNT hybrids immediately prompted us to explore structure-based sorting of CNTs $[5,6]$. The phosphate groups on a DNA-CNT hybrid render a negative charge density on the $\mathrm{CNT}$, the magnitude of which should be a function of the DNA sequence and the electronic properties of the tube. In order to take advantage of such differences for CNT separation, we explored ion-exchange liquid chromatography (IEX). This is a separation technique widely used in chemical and biochemical labs, and in the pharmaceutical industry and is a controlled process of adsorption and desorption of the target molecules on charged surfaces (Scheme 1). The outcome of anionexchange-based DNA-CNT separation, as measured by optical absorption spectral changes from fraction to

\section{DNA-CNT}
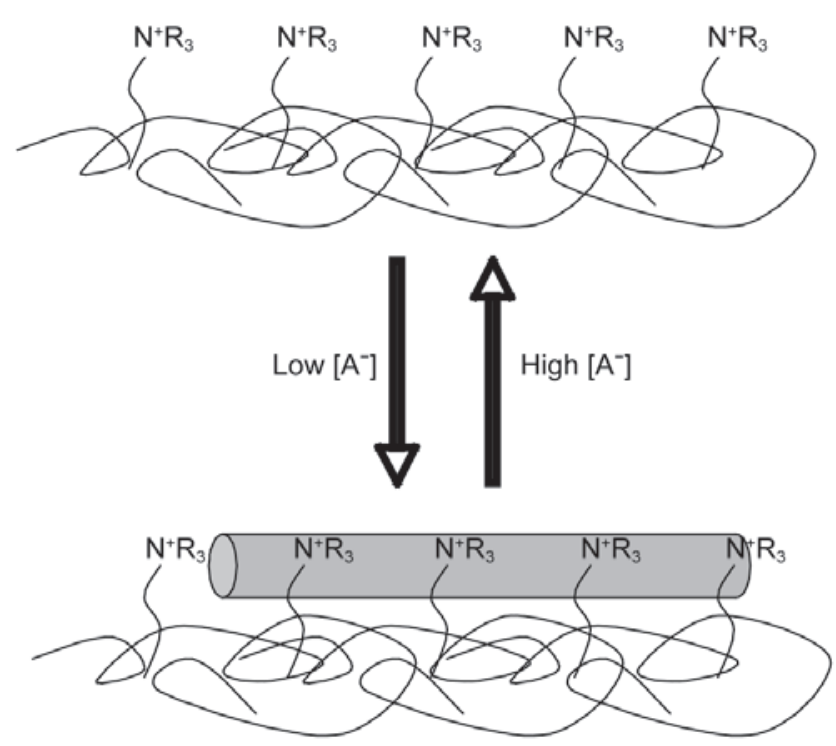

Scheme 1 Anion exchange separation of DNA-CNTs. Anion exchange columns are typically packed with micro-sized polymer or silica beads that are surface-grafted with positively charged functional groups such as quaternary amines. DNA-CNTs injected into a column can be either adsorbed on the beads or remain free, depending on the concentration of anionic species $\left(A^{-}\right)$in the mobile phase. The equilibrium is also dependent on CNT structure, leading to fractionation of DNA-CNTs when they are eluted from the column by a salt gradient

fraction, is strongly dependent on the DNA sequence. Through a systematic but limited search of the ssDNA library under identical chromatographic conditions, the best separation was obtained with a sequence of alternating guanine $(\mathrm{G})$ and thymine $(\mathrm{T})$ nucleotides, $(\mathrm{GT})_{n}$, with total length ranging from 20 to 90 bases ( $n$ $=10$ to 45$)$ [6]. 


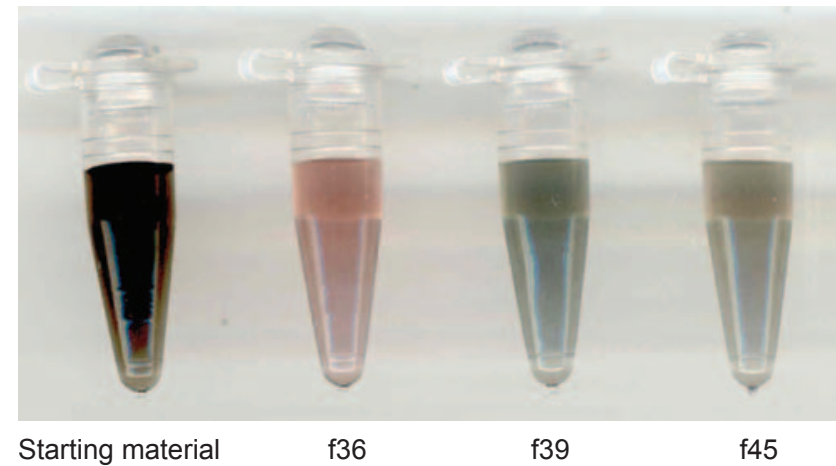

(a)

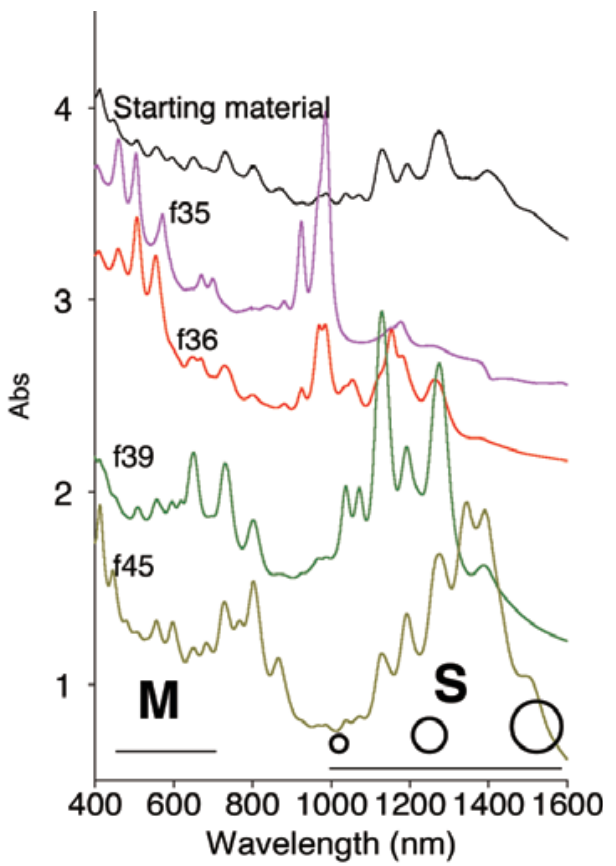

(b)

Figure 3 (a) IEX fractions obtained using $(\mathrm{GT})_{30}$ dispersed HiPCO nanotubes as the starting material; (b)UV-vis-near IR spectra of the corresponding IEX fractions

Chromatography is a century old technique. The name reflects its original use in separating species of different color. In the case of high-pressure carbon monoxide (HiPCO) CNTs, a visual comparison of the black starting material with fractionated materials of different color suggests immediately that the CNTs have been sorted according to their electronic structures (Fig. 3(a)). Absorption spectroscopy reveals a more quantitative analysis of the separation. Figure $3(\mathrm{~b})$ shows the UV-vis-near IR absorption spectra of fractionated $(\mathrm{GT})_{30}$-CNTs. Three regions are identified in Fig. 3(b): first inter-band transitions for metals, $\mathrm{M}_{11}(400-650 \mathrm{~nm})$; and first and second inter-band transitions for semiconductors, $S_{11}(900-$
$1600 \mathrm{~nm})$ and $S_{22}(550-900 \mathrm{~nm})$. The starting material yields a spectrum typical of singly dispersed CNTs in aqueous solution, with multiple peaks arising from different types of CNTs overlapping across the entire spectrum. In contrast, the spectrum from an early faction $\mathrm{f} 35$ has only one major peak centred at $980 \mathrm{~nm}$ in the $S_{11}$ region, corresponding to the $S_{11}$ transition from the smallest diameter semiconducting tubes found in HiPCO CNTs. Additionally $\mathrm{M}_{11}$ transitions are enhanced, indicating enrichment in metallic tubes. The $\mathrm{S}_{11}$ region from later fractions ( $\mathrm{f} 36, \mathrm{f} 39$, and f45) shows a systematic shift of intensity towards longer wavelength. Since, to a first approximation, the $S_{11}$ transition wavelength is proportional to the tube diameter, the observed shift indicates a gradual increase in average semiconducting tube diameter from early to late fractions. There is also a decrease in the $\mathrm{M}_{11}$ intensities in late fractions, corresponding to a depletion of metallic tubes.

The simultaneous processes of both metal/ semiconductor and diameter-based separation shown above are not desirable if pure metallic fractions are needed. Decoupling of the two processes can be achieved by controlling the length of DNA: enrichment of metallic tubes becomes more effective as the length of DNA decreases. Figure 4 shows metal/semiconductor separation of laser-ablation tubes dispersed by $(\mathrm{GT})_{10}$. In this case, the metallic tube-enriched fraction has minimum contamination by small diameter semiconducting tubes.

In parallel to the structure-based separation,

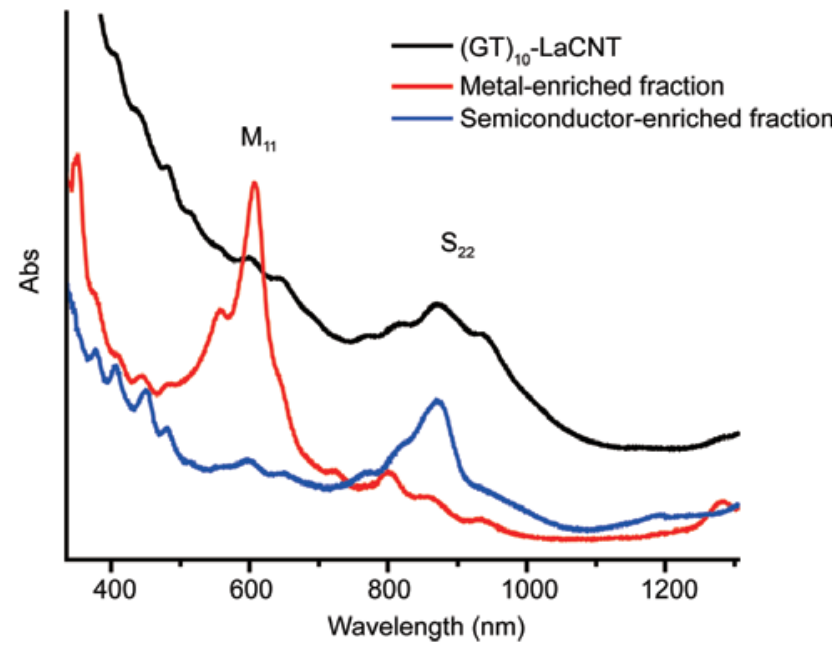

Figure 4 UV-vis-near IR spectra of IEX separated $(\mathrm{GT})_{10}$ dispersed laser ablation nanotubes (LaCNT)

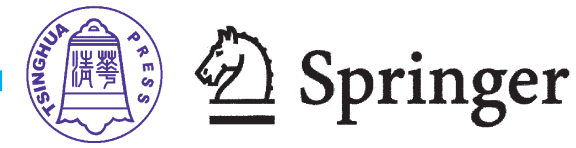


effective nanotube length separation using conventional size-exclusion chromatography (SEC) has also been developed [19]. In collaboration with Sepax Technologies, we identified a type of silicabased column resin that shows minimum adsorption of DNA-CNT. Three such columns in series with pore sizes of $2000 \AA, 1000 \AA$, and $300 \AA$, were found to separate DNA-CNT into fractions of very narrow length distribution, as measured directly by AFM. The average length decreases monotonically from $>500 \mathrm{~nm}$ in the early fractions to $<100 \mathrm{~nm}$ in the late fractions, with length variation $\leqslant 10 \%$ in each of the measured fractions. Using UV-visnear IR spectroscopy, we also showed that SEC is very effective in removing graphitic impurities that contribute to the optical absorption spectral baseline and a broad peak at $\sim 270 \mathrm{~nm}$.

The ability to control tube length enabled us to improve IEX separation of DNA-CNTs. Ultrasonically dispersed CNTs are randomly cut during the process, resulting in tubes ranging from 50 to $1000 \mathrm{~nm}$ in length. To a first approximation, the electronic structure of a CNT is not dependent on its length. However, length becomes a relevant factor when tubes undergo differential movement driven by an external field or flow pressure during a separation process. A broad length distribution inevitably reduces separation resolution. Indeed, a much improved IEX separation of small diameter CNTs was obtained when a prior SEC separation was used to narrow down the length distribution. This combination allowed purification of a few single chirality enriched CNTs using tubes prepared by the CoMoCAT catalytic process as the starting material [7].

Figure 5(a) shows optical absorption spectra of fractions that are enriched in $(6,4),(9,1)$, and $(6,5)$ tubes, respectively. The AFM image in Fig. 5(b) illustrates the purity and length distribution of a representative $(6,5)$ enriched fraction. The $(6,5)$ tubes represent the most abundant species in the CoMoCAT material. We have previously reported the purification of $(6,5)$ tubes by IEX alone [30]. The $(6,5)$ fraction obtained by the two chromatography steps appears to be more pure. Its spectrum not only shows $\mathrm{E}_{11}(991 \mathrm{~nm})$ and $\mathrm{E}_{22}(574 \mathrm{~nm})$ transitions, but also reveals an $E_{33}$ transition at $348 \mathrm{~nm}$. The $(9,1)$

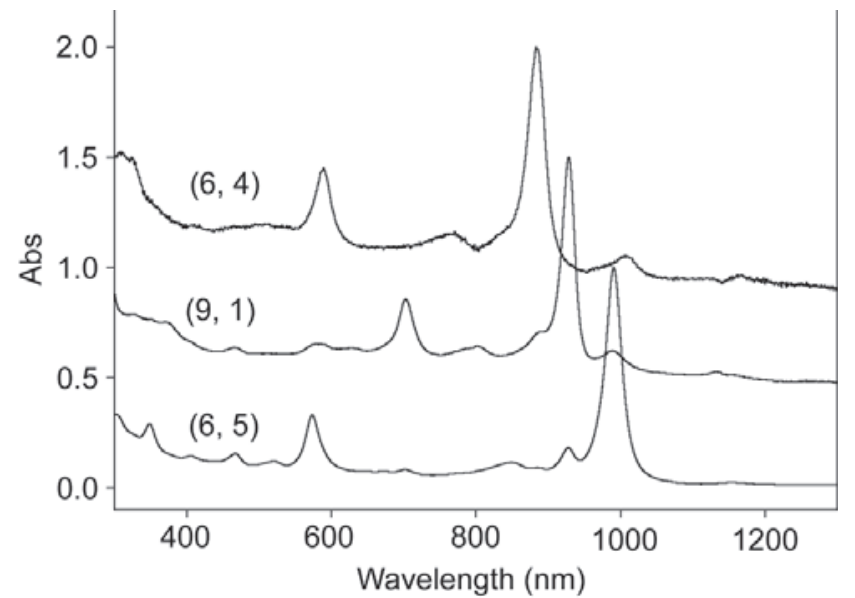

(a)

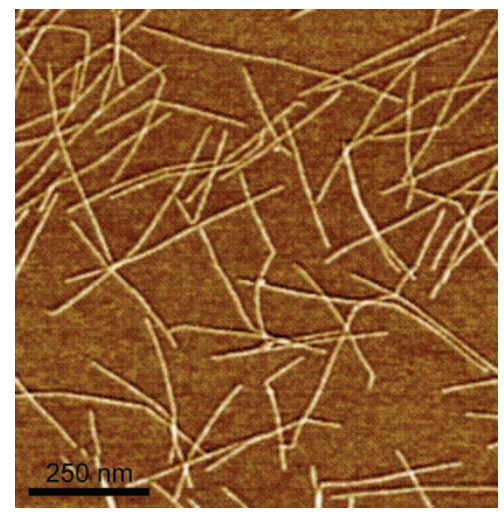

(b)

Figure 5 (a) UV-vis-near IR spectra of single chirality enriched fractions obtained by two step (SEC + IEX) chromatography; (b) AFM image of $(6,5)$ enriched fraction (From Ref. [7], used with permission)

tubes are identical to $(6,5)$ in diameter $(0.757 \mathrm{~nm})$ but have different chirality (Fig. 1(a)). Their $E_{11}$ and $E_{22}$ transitions occur at $928 \mathrm{~nm}$ and $703 \mathrm{~nm}$, respectively. The $(6,4)$ fraction has two prominent peaks at 883 $\mathrm{nm}$ and $589 \mathrm{~nm}$, corresponding to the $E_{11}$ and $E_{22}$ transitions. We should point out that there are large differences in the abundance of the three tubes in the starting CoMoCAT material; the $(6,4)$ and $(9,1)$ tubes are estimated to be fewer in number than $(6,5)$ by factors of 100 and 20, respectively. Purification of $(9,1)$ from the much more abundant $(6,5)$ species with the same diameter but different chiralities, thus clearly demonstrates the potential of the DNA-based approach.

\section{Mechanisms of separation}

Understanding the mechanism of IEX separation 
is an on-going effort that may ultimately lead to predicting DNA wrapping structures, and devising other more effective ways for separation. Any mechanistic proposal has to be able to explain the marked dependence of IEX separation on DNA sequence. To explain the unique features of the (GT) sequence responsible for the separation, we proposed a model for the $(\mathrm{GT})_{n}$-CNT structure, in which two anti-parallel (GT) $)_{n}$ strands interact with each other through hydrogen bonds to form a double-stranded strip, which then wraps around the CNT with closepacked bases resembling molecular tiles lying on the side-wall of the nanotube [6]. Such a doublehelical structure is built on the unique hydrogen bonding network between two $(\mathrm{GT})_{n}$ strands, and is expected to narrow down the allowed conformations for the wrapping DNA. This should lead to a more defined charge density and narrower elution profile for a given $(n, m)$ tube, and eventually successful separation. This is probably why most other DNA sequences disperse CNTs but do not give good separation. Our proposed $(\mathrm{GT})_{n}$ structure model was examined by Johnson et al., and was found to be sterically rather unfavourable, however [25]. While the specific structure we proposed may not be correct, some kind of inter-strand interaction is likely to be needed in order to explain the unique features of the (GT) $)_{n}$ wrapping.

Current understanding of the separation mechanism is still largely based on the electrostatics of the DNA-CNT hybrid and its interaction with the positively charged substrate of the anion exchange resin (Scheme 1) [31]. The DNA-CNT hybrid carries negative charges because of the deprotonated backbone phosphate groups on the DNA. The interactions of the hybrid with the positively charged anion-exchange resin and the eluting salt solution are electrostatic in nature, and depend on the linear charge density of the hybrid. The effective net linear charge density of the hybrid is mainly determined by the linear charge density of the phosphate groups along the nanotube axis. This value is modulated by differences in the electronic character of the nanotube core. For metallic tubes, the discrete negative charges on the DNA create an electrostatic field along the tube axis, which induces positive screening image charges in the nanotube. As a result, the net linear charge density of the DNA-CNT hybrid is reduced from that of the DNA wrap alone. For semiconducting tubes, the lower polarizability of the nanotube, compared to that of the surrounding water, results in an increased effective linear charge density of the DNA-CNT hybrid relative to that of the DNA wrap alone, based on an image charge analysis for adjacent dielectrics. This fundamental difference in behavior provides ample differentiation between the binding strengths of metallic and semiconducting nanotubes to the anion exchange resin. For semiconducting DNA-CNTs, there are two (non-exclusive) mechanisms responsible for the dependence of linear charge density on tube diameter. First, because the polarizability of semiconducting nanotubes depends on diameter, the effective linear charge density is diameter-dependent. Second, the linear charge density of the DNA can change with tube diameter due to wrapping geometry changes. Together, these allow diameter-dependent separation of semiconducting tubes.

Enrichment of $(9,1)$, in the presence of the more abundant $(6,5)$ tubes with the same diameter and electronic type, is most difficult to understand. Such exquisite separation must be the result of chiralitydependent interactions between DNA-wrapped CNTs and the IEX resin. These interactions could be electrostatic in nature, arising from chirality-dependent DNA-wrapping [32] and/or electrodynamic in nature, originating from chirality-dependent van der Waals forces. Indeed, the requirement that chaotropic salts be present for the IEX elution suggests that van der Waals interactions play a role.

\section{Path forward}

So far, we have shown the capability of the DNAbased approach in metal/semiconductor CNT separation, and single chirality CNT enrichment for certain small diameter tubes. The latter demonstrates the exquisite resolution power of the DNA approach. In addition to our own work, others have also employed IEX and SEC methods for DNA-CNT separation [4, 33-35]. The separated DNA-CNTs have found use in both fundamental studies [24, 30, 36-43] and applications $[35,44]$. In comparison with other 
separation approaches, chromatographic separation of DNA-CNTs offers higher resolution and requires shorter processing times.

In general, liquid chromatography is a scalable process routinely employed in the biotech and pharmaceutical industries. Scaling up the chromatographic separation of DNA-CNTs requires tackling a few technical issues. The first is the cost of DNA. At $\$ 10 / \mathrm{mg}$, customer oligonucleotides are too expensive to be used in large scale separations. However, we do not view this as an insurmountable problem. Solid-phase DNA synthesis is a mature technology. The business model of current DNA oligonucleotide suppliers is designed to meet the needs of molecular biologists who order ultra-pure oligonucleotides, in small amounts and with different sequences from time to time. A large portion of the cost is from instrument setting up, DNA purification, and express shipping and handling. These cost factors do not exist for large-scale synthesis of a fixed sequence with low purity requirement. Ultimately, the economics of the scale-up of DNA-CNT separation will be determined by the nature of end uses.

Another issue related to the scale-up is the separation efficiency of IEX columns. A column resin is typically composed of micro-sized polymer beads or silica, with a surface coating of charged functional groups (Scheme 1). Commercially available IEX columns suitable for DNA-CNT separation are not designed for the purpose. As a result, these columns have low recovery $(\sim 30 \%)$, which limits separation yield and column life-time. A number of column-related factors strongly influence adsorption and desorption of the DNA-CNT hybrids. These include chemical identity, and surface density of the positively charged ion-exchange groups as well as the morphology and hydrophobicity/hydrophilicity of the core surface on which the ion-exchange groups are grafted. A systematic optimization of these parameters could potentially lead to dramatic improvements in both separation resolution and recovery.

Understanding the mechanisms of the DNAbased separation approach is of both scientific and technological significance. At the heart of the problem is the structure of DNA-CNT. Experimental evidence strongly suggests that the hybrid structure is dependent on both DNA sequence and CNT structure. So far, the structural information comes primarily from low resolution AFM obtained from dried samples. Whether or not it is relevant to the solution state structure is an open question. High resolution TEM and solution-phase techniques such as circular dichroism may provide a more accurate measure of the structure. It is encouraging that molecular dynamics-based theoretical approaches are starting to provide quantitative description of both the thermodynamics and kinetics of the DNA wrapping process $[25,45]$. The DNA-CNT hybrid presents a unique challenge in theoretical modeling studies. How to incorporate the band structure into the force field? How to effectively deal with water and salt molecules? These are important questions waiting to be addressed. It is our hope that the DNACNT hybrid may serve as a model system for the further development of molecular dynamics for macromolecules in general.

Are there better DNA sequences for CNT separation? Current solid-phase synthesis can produce $\sim 100$-base long oligonucelotides. The size of the ssDNA library available to us is therefore on the order of $4^{100}$. Given such an astronomical size, it is reasonable to expect that there exist other sequences for better CNT separation. What is needed is a rational search strategy, based either on predictions of molecular dynamics modeling, or on some ingenious experimental design, in order to discover these sequences. For a given sequence composition, the length may also play a very important role, as hinted by the improved metal/semiconductor separation with shorter $(\mathrm{GT})_{n}$ sequences. This generates another whole dimension of opportunity for DNA-CNT interactions. We and our collaborators are currently searching for better sequences and examining length effects. It is hoped that the outcome of this effort will greatly expand our molecular tool box for CNT manipulation, shed new light on the mechanisms by which DNA-based separation takes place, and provide new molecular recognition concepts to the field of supramolecular chemistry. 


\section{Achnowledgements}

Work conducted in our lab is supported by DuPont and US National Science Foundation. We would also like to acknowledge our collaborators Anand Jagota, Ellen Semke, Timothy Devine, and Robert S. Mclean, for their contribution to the CNT separation work presented in this review.

\section{References}

[1] Saito, R.; Dresselhaus, G.; Dresselhaus, M. S. Physical Properties of Carbon Nanotubes; Imperial College Press: London, 1999.

[2] Bachilo, S. M.; Balzano, L.; Herrera, J. E.; Pompeo, F.; Resasco, D. E.; Weisman, R. B. Narrow $(n, m)$-distribution of single-walled carbon nanotubes grown using a solid supported catalyst. J. Am. Chem. Soc. 2003, 125, 1118611187.

[3] Ciuparu, D.; Chen, Y; Lim, S; Haller, G. L.; Pfefferle, L. Uniform-diameter single-walled carbon nanotubes catalytically grown in cobalt-incorporated MCM-41. J. Phys. Chem. B 2004, 108, 503-507.

[4] Li, X.; Tu, X.; Zaric, S.; Welsher, K.; Seo, W. S.; Zhao, W.; Dai, H. Selective synthesis combined with chemical separation of single-walled carbon nanotubes for chirality selection. J. Am. Chem. Soc. 2007, 129, 1577015771.

[5] Zheng, M.; Jagota, A.; Semke, E. D.; Diner, B. A.; McLean, R. S.; Lustig, S. R.; Richardson, R. E.; Tassi, N. G. DNA-assisted dispersion and separation of carbon nanotubes. Nat. Mater. 2003, 2, 338-342.

[6] Zheng, M.; Jagota, A.; Strano, M. S.; Santos, A. P.; Barone, P.; Chou, S. G.; Diner, B. A.; Dresselhaus, M. S.; McLean, R. S.; Onoa, G. B.; Samsonidze, G. G.; Semke, E. D.; Usrey, M.; Walls, D. J. Structure-based carbon nanotube sorting by sequence-dependent DNA assembly. Science 2003, 302, 1545-1548.

[7] Zheng, M.; Semke, E. D. Enrichment of single chirality carbon nanotubes. J. Am. Chem. Soc. 2007, 129, 60846085.

[8] Chen, Z.; Du, X.; Du, M.; Rancken, C. D.; Cheng, H.; Rinzler, A. G. Bulk separative enrichment in metallic or semiconducting single-walled carbon nanotubes. Nano. Lett. 2003, 3, 1245-1249.

[9] Chattopadhyay, D.; Galeska, I.; Papadimitrakopoulos,
F. A route for bulk separation of semiconducting from metallic single-wall carbon nanotubes. J. Am. Chem. Soc. 2003, 125, 3370-3375.

[10] Krupke, R.; Hennrich, F.; Löhneysen, H. V.; Kappes, M. $M$. Separation of metallic from semiconducting singlewalled carbon nanotubes. Science 2003, 301, 344-347.

[11] Arnold, M. S.; Stupp, S. I.; Hersam, M. C. Enrichment of single-walled carbon nanotubes by diameter in density gradient. Nano Lett. 2005, 5, 713-718.

[12] Maeda, Y.; Kimura, S. I.; Kanda, M.; Hirashima, Y.; Hasegawa, T.; Wakahara, T.; Lian, Y.; Nakahodo, T.; Tsuchiya, T.; Akasaka, T.; et al. Large-scale separation of metallic and semiconducting single-walled carbon nanotubes. J. Am. Chem. Soc. 2005, 127, 10287-10290.

[13] Heller, D.A.; Mayrhofer, R. M.; Baik, S.; Grinkova, Y. V.; Usrey, M. L.; Strano, M. S. Concomitant length and diameter separation of single-walled carbon nanotubes. J. Am. Chem. Soc. 2004, 126, 14567-14573.

[14] Arnold, M.S.; Green, A. A.; Hulvat, J. F.; Stupp, S. I.; Hersam, M. C. Sorting carbon nanotubes by electronic structure using density differentiation. Nat. Nanotechnol. 2006, 1, 60-65.

[15] Peng, H.; Alvarez, N. T.; Kittrell, C.; Hauge, R. H.; Schmidt, H. K. Dielectrophoresis field flow fractionation of single-walled carbon nanotubes. J. Am. Chem. Soc. 2006, 128, 8396-8397.

[16] Kim, W. J.; Usrey, M. L.; Strano, M. S. Selective functionalization and free solution electrophoresis of single-walled carbon nanotubes: Separate enrichment of metallic and semiconducting SWNT. Chem. Mater. 2007, 19, 1571-1576.

[17] Weisman, R. B. Four degrees of separation. Nat. Mater. 2003, 2, 569-570.

[18] Hersam, M. C. Progress towards monodisperse singlewalled carbon nanotubes. Nat. Nanotechnol. 2008, 3, 387-394.

[19] Huang, X.; McLean, R. S.; Zheng, M. High-resolution length sorting and purification of DNA-wrapped carbon nanotubes by size-exclusion chromatography. Anal. Chem. 2005, 77, 6225-6228.

[20] Tao, N. J.; Shi, Z. Monolayer guanine and adenine on graphite in $\mathrm{NaCl}$ solution: A comparative STM and AFM study. J. Phys. Chem. B 1994, 98, 1464-1471.

[21] Sowerby, S. J.; Edelwirth, M.; Heckl, W. M. Self-assembly at the prebiotic solid-liquid interface: Structure of selfassembled monolayers of adenine and guanine bases 
formed on inorganic surfaces. J. Phys. Chem. B 1998, 102, 5914-5922.

[22] Sowerby, S. J.; Cohn, C. H.; Heckl, W. M.; Holm, N. G. Differential adsorption of nucleic acid bases: Relevance to the origin of life. Proc. Natl. Acad. Sci. U. S. A, 2001, 98, 820-822.

[23] Wilson, D. S. ; Szostak, J. W. In vitro selection of functional nucleic acids. Annu. Rev. Biochem.1999, 68, 611-647.

[24] Dukovic, G.; Balaz, M.; Doak, P.; Berova, N. D.; Zheng, M.; McLean, R. S.; Brus, L. E. Racemic single-walled carbon nanotubes exhibit circular dichroism when wrapped with DNA. J. Am. Chem. Soc. 2006, 128, 9004-9005.

[25] Johnson, R. R.; Johnson, A. T. C. ; Klein, M. L. Probing the structure of DNA-carbon nanotube hybrids with molecular dynamics. Nano Lett. 2008, 8, 69-75.

[26] Enyashin, A. N.; Gemming, S.; Seifert, G. DNA-wrapped carbon nanotubes. Nanotechnology 2007, 18, 245702.

[27] Frischknecht, A. L.; Martin, M. G. Simulation of the adsorption of nucleotide monophosphates on carbon nanotubes in aqueous solution. J. Phys. Chem. C 2008, 112, 6271-6278.

[28] Gowtham, S.; Scheicher, R. H.; Pandey, R.; Karna, S.; Ahuja, R. First-principles study of physisorption of nucleic acid bases on small-diameter carbon nanotubes. Nanotechnology 2008, 19,125701.

[29] Meng, S.; Maragakis, P.; Papaloukas, C.; Kaxiras, E. DNA nucleoside interaction and identification with carbon nanotubes. Nano Lett. 2007, 7, 45-50.

[30] Zheng, M.; Diner, B. A. Solution redox chemistry of carbon nanotubes. J. Am. Chem. Soc. 2004, 126, 15490-15494.

[31] Lustig, S. R.; Jagota, A.; Khripin, C.; Zheng, M. Theory of structure-based carbon nanotube separations by ionexchange chromatography of DNA/CNT hybrids. J. Phys. Chem. B 2005, 109, 2559-2566.

[32] Wei, C. Radius and chirality dependent conformation of polymer molecule at nanotube interface. Nano Lett. 2006, 6, 1627-1631.

[33] Bauer, B. J.; Becker, M. L.; Bajpai, V.; Fagan, J. A.; Hobbie, E. K.; Migler, K.; Guttman, C. M.; Blair, W. R. Measurement of single-wall nanotube dispersion by size exclusion chromatography. J. Phys. Chem. C 2007, 111, 17914-17918.

[34] Bauer, B.J.; Fagan, J. A.; Hobbie, E. K.; Chun, J.; Bajpai, V. Chromatographic fractionation of SWCNT/DNA dispersions with on-line multi-angle light scattering. J.
Phys. Chem. C 2008, 112, 1842-1850.

[35] Zhang, L.; Zaric, S.; Tu, X.; Wang, X.; Zhao, W.; Dai, H. Assessment of chemically separated carbon nanotubes for nanoelectronics. J. Am. Chem. Soc. 2008, 130, 26862691.

[36] Chou, S. G.; Decamp, M. F.; Jiang, J.; Samsonidze, G. G.; Barros, E. B.; Plentz, F.; Jorio, A.; Zheng, M.; Onoa, G. B.; Semke, E. D.; et al. Phonon-assisted exciton relaxation dynamics for a $(6,5)$-enriched DNA-wrapped single-walled carbon nanotube sample. Phys. Rev. B 2005, 72, 195415.

[37] Chou, S. G.; Plentz, F.; Jiang, J.; Saito, R.; Nezich, D.; Ribeiro, H. B.; Jorio, A.; Pimenta, M. A.; Samsonidze, G. G.; Santos, A. P.; et al. Phonon-assisted excitonic recombination channels observed in DNA-wrapped carbon nanotubes using photoluminescence spectroscopy. Phys. Rev. Lett. 2005, 94, 127402.

[38] Torrens, O. N.; Milkie, D. E.; Zheng, M.; Kikkawa, J. M. Photoluminescence from intertube carrier migration in single-walled carbon nanotube bundles. Nano Lett. 2006, 6, 2864-2867.

[39] Zheng, M.; Rostovtsev, V. V. Photoinduced charge transfer mediated by DNA-wrapped carbon nanotubes. J. Am. Chem. Soc. 2006, 128, 7702-7703.

[40] Carlson, L. J.; Maccagnano, S. E.; Zheng, M.; Silcox, J.; Krauss, T. D. Fluorescence efficiency of individual carbon nanotubes. Nano Lett. 2007, 7, 3698-3703.

[41] Chou, S. G.; Son, H.; Kong, J.; Jorio, A. Length characterization of DNA-wrapped carbon nanotubes using Raman spectroscopy. Appl. Phys. Lett. 2007, 90, 131109.

[42] Torrens, O. N.; Milkie, D. E.; Ban, H. Y.; Zheng, M.; Onoa, G. B.; Gierke, T. D.; Kikkawa, J. M. Measurement of chiral-dependent magnetic anisotropy in carbon nanotubes. J. Am. Chem. Soc. 2007, 129, 252-253.

[43] Song, D.; Wang, F.; Dukovic, G.; Zheng, M.; Semke, E. D.; Brus, L. E.; Heinz, T. F. Direct measurement of the lifetime of optical phonons in single-walled carbon nanotubes. Phys. Rev. Lett. 2008, 100, 225503.

[44] Becker, M. L.; Fagan, J. A.; Gallant, N. D.; Bauer, B. J.; Bajpai, V.; Hobbie, E. K.; Lacerda, S. H.; Migler, K. B.; Jakupciak, J. P. Length-dependent uptake of DNAwrapped single-walled carbon nanotubes. Adv. Mater. 2007, 19, 939-945.

[45] Manohar, S.; Tang, T.; Jagota, A. Structure of homopolymer DNA-CNT hybrids. J. Phys. Chem. C 2007, $111,17835-17845$. 